\title{
On the Statistics of Cognitive Radio Capacity in Shadowing and Fast Fading Environments
}

\author{
Muhammad Fainan Hanif *, Peter J. Smith ${ }^{*}$ and Mansoor Shafi ${ }^{\dagger}$ \\ *Department of Electrical and Computer Engineering, University of Canterbury, Christchurch, New Zealand \\ $\dagger$ Telecom New Zealand, PO Box 293, Wellington, New Zealand \\ Email:mfh21@student.canterbury.ac.nz,p.smith@elec.canterbury.ac.nz, mansoor.shafi@telecom.co.nz
}

\begin{abstract}
In this paper we consider the capacity of the cognitive radio $(\mathrm{CR})$ channel in a fading environment under a "low interference regime". This capacity depends critically on a power loss parameter, $\alpha$, which governs how much transmit power the CR dedicates to relaying the primary message. We derive a simple, accurate approximation to $\alpha$ which gives considerable insight into system capacity. We also investigate the effects of system parameters and propagation environment on $\alpha$ and the CR capacity. In all cases, the use of the approximation is shown to be extremely accurate. Finally, we derive the probability that the "low interference regime" holds and demonstrate that this is the dominant case, especially in practical CR deployment scenarios.
\end{abstract}

\section{INTRODUCTION}

The key idea behind the deployment of cognitive radio (CR) is that greater utilization of spectrum can be achieved if they are allowed to co-exist with the incumbent licensed primary users (PUs) provided that they cause minimal interference. The CRs must therefore learn from the radio environment and adapt their parameters so that they can co-exist with the primary systems. The CR field has proven to be a rich source of challenging problems. A large number of papers have appeared on various aspects of CR, namely spectrum sensing [1], fundamental limits of spectrum sharing [2], information theoretic capacity limits [3]-[6] etc.

The 2 user cognitive channel [3]-[6] consists of a primary and a secondary user. It is very closely related to the classic 2 user interference channel, see [7] and references therein.

The formulation of the CR channel is due to Devroye et al. [3]. In this channel, the CR has a non-causal knowledge of the intended message of the primary and by employing dirty paper coding [8] at the CR transmitter it is able to circumvent the primary user's interference to its receiver. However, the interference from the $\mathrm{CR}$ to the primary receiver remains and has the potential to cause a rate loss to the primary.

In recent work, Jovicic and Viswanath [5] have studied the fundamental limits of the capacity of the CR channel. They show that if the CR is able to devote a part of its power to relaying the primary message, it is possible to compensate for the rate loss to the primary via this additional relay. They have provided exact expressions for the PU and CR capacity of a 2 user CR channel when the CR transmitter sustains a power loss by devoting a fraction, $\alpha$, of its transmit power to relay the PU message. Furthermore, they have provided an exact expression for $\alpha$ such that the PU rate remains the same as if there was no CR interference. It should be stressed here that their system model is such that at the expense of CR transmit power, the PU device is always able to maintain a constant data rate. Hence, we focus on CR rate, $\alpha$ and their statistics. They also assume that the PU receiver uses a single user decoder. Their result holds for the so called low interference regime when the received SNR of the CR transmission is lesser at the primary receiver (i.e., interference from $\mathrm{CR}$ to $\mathrm{PU}$ ) than at the CR receiver. The authors in [9] also arrived at the same results in their parallel but independent work.

The Jovicic and Viswanath study is for a static channel, i.e., the direct and cross link gains are constants. In a system study, these gains will be random and subject to distance dependent path loss and shadow fading. Furthermore, the channel gains also experience fast fading. As the channel gains are random variables, the power loss parameter, $\alpha$, is also random.

In this paper we focus on the power loss, $\alpha$, the capacity of the CR channel and the probability that the "low interference regime" holds. The motivation for this work arises from the fact that maximum rate schemes for the $\mathrm{CR}$ in the low interference regime [5] and the achievable rate schemes for the high interference regime [4], [6] are very different. Hence, it is of interest to identify which scenario is the most important. To attack this question we propose a simple, physically based geometric model for the CR, PU layout and compute the probability of the low interference regime. Results are obviously limited to this particular model but provide some insight into reasonable deployment scenarios. Since the results show the low interference regime can be dominant, it is also of interest to characterize CR performance via the $\alpha$ parameter. In this area we make the following contributions:

- Assuming lognormal shadowing, Rayleigh fading and path loss effects we derive the probability that the "low interference regime" holds.

- In the same fading environment we derive an approximation for $\alpha$ and its statistics. This extremely accurate approximation leads to simple interpretations of the effect of system parameters on the capacity.

- Using the statistics of $\alpha$ we investigate the mean rate loss of the $\mathrm{CR}$ and the cumulative distribution function (CDF) of the CR rates. For both the above we show their dependence on the propagation parameters.

- We also show how the mean value of $\alpha$ varies with the $\mathrm{CR}$ transmit power and therefore the CR coverage area. 
This paper is organized as follows: Section II describes the system model. Section III derives the probability that the "low interference regime" holds and in Section IV an approximation for $\alpha$ is developed. Section V presents analytical and simulation results and some conclusions are given in Section VI.

\section{SySTEM MODEL}

Consider a PU receiver in the center of a circular region of radius $R_{p}$. The PU transmitter is located uniformly in an annulus of outer radius $R_{p}$ and inner radius $R_{0}$ centered on the PU receiver. It is to be noted that we place the PU receiver at the center only for the sake of mathematical convenience (see Fig. 1). The use of the annulus restricts devices from being too close to the receiver. This matches physical reality and also avoids problems with the classical inverse power law relationship between signal strength and distance [10]. In particular, having a minimum distance, $R_{0}$, prevents the signal strength from becoming infinite as the transmitter approaches the receiver. Similarly, we assume that a CR receiver is uniformly located in the same annulus. Finally, a CR transmitter is uniformly located in an annulus centered on the CR receiver. The dimensions of this annulus are defined by an inner radius, $R_{0}$, and an outer radius, $R_{c}$. Following the work of Jovicic and Viswanath [5], the four channel gains which define the system are denoted $p, g, f, c$. In this paper, these complex channel gains include shadow fading, path-loss and Rayleigh fast fading effects. To introduce the required notation we consider the link from the $\mathrm{CR}$ transmitter to the PU receiver, the CP link. For this link we have:

$$
|f|^{2}=\Gamma_{c p}|\tilde{f}|^{2},
$$

where $|\tilde{f}|^{2}$ is an exponential random variable with unit mean and $\Gamma_{c p}$ is the link gain. The link gain comprises shadow fading and distance dependent path loss effects so that,

$$
\Gamma_{c p}=A_{c} L_{c p} r_{c p}^{-\gamma},
$$

where $A_{c}$ is a constant, $L_{c p}=10^{\tilde{X}_{c p} / 10}$ is lognormal, $\tilde{X}_{c p}$ is zero mean Gaussian and $r_{c p}$ is the link distance. The standard deviation which defines the lognormal is $\sigma(\mathrm{dB})$ and $\gamma$ is the path loss exponent. For convenience, we also write $L_{c p}=e^{X_{c p}}$ so that $X_{c p}=\beta \tilde{X}_{c p}, \beta=\ln (10) / 10$ and $\sigma_{s f}^{2}$ is the variance of $X_{c p}$. Hence, for the CP link we have:

$$
|f|^{2}=A_{c} e^{X_{c p}} r_{c p}^{-\gamma}|\tilde{f}|^{2} .
$$

The other three links are defined similarly where $\tilde{p}, \tilde{g}, \tilde{c}$ are standard exponentials, $X_{p p}, X_{p c}, X_{c c}$, are Gaussians with the same parameters as $X_{c p}$ and $r_{p p}, r_{p c}, r_{c c}$ are link distances. However, for the links involving PU transmitter we assume a constant $A_{p}$ in the model of link gains. The parameters $A_{p}$ and $A_{c}$ are constant and all links are assumed independent. The remaining parameters required are the transmit powers of the PU/CR devices, given by $P_{p} / P_{c}$, and the noise powers at the PU/CR receivers, given by $N_{p} / N_{c}$.

For fixed channel coefficients, $p, g, f$ and $c$, Jovicic and Viswanath [5] compute the highest rate that the CR can achieve subject to certain constraints. A key constraint is that the PU must not suffer any rate degradation due to the CR and

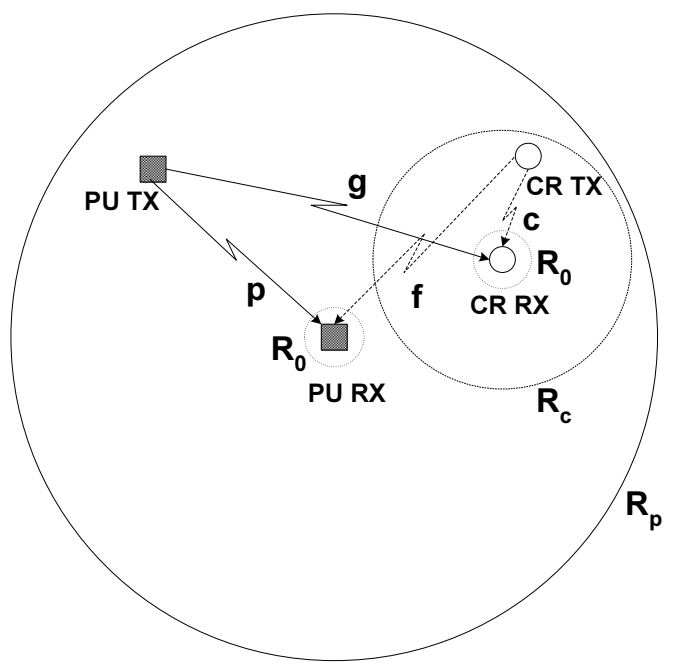

Fig. 1. System model.

this is achieved by the CR dedicating a portion, $\alpha$, of its transmit power to relaying the PU message. The parameter, $\alpha$, is therefore central to determining the CR rate. Furthermore, the results in [5] are valid in the "low interference regime" defined by $a<1$ where:

$$
a=\frac{\sqrt{N_{c}} \sqrt{\Gamma_{c p}}|\tilde{f}|}{\sqrt{N_{p}} \sqrt{\Gamma_{c c}}|\tilde{c}|}=\frac{\sqrt{N_{c}} e^{X_{c p} / 2} r_{c p}^{-\gamma / 2}|\tilde{f}|}{\sqrt{N_{p}} e^{X_{c c} / 2} r_{c c}^{-\gamma / 2}|\tilde{c}|} .
$$

In this regime, the highest $\mathrm{CR}$ rate is given by

$$
R_{C R}=\log _{2}\left(1+\frac{|c|^{2}(1-\alpha) P_{c}}{N_{c}}\right),
$$

with the power loss parameter, $\alpha$, defined by

$$
\alpha=\frac{|s|^{2}}{|t|^{2}}\left[\frac{\sqrt{1+|t|^{2}\left(1+|s|^{2}\right)}-1}{1+|s|^{2}}\right]^{2},
$$

where $|s|=\sqrt{P_{p}} \sqrt{\Gamma_{p p}}|\tilde{p}| N_{p}^{-1 / 2}$ and $|t|=$ $\sqrt{P_{c}} \sqrt{\Gamma_{c p}}|\tilde{f}| N_{p}^{-1 / 2}$. Note that the definitions of $\alpha$ and $R_{c}$ are conditional on $a<1$. Since $a$ is a function of $\tilde{f}$ and $\tilde{c}$ we see that both $\tilde{f}$ and $\tilde{c}$ are conditional exponentials.

\section{THE LOW INTERFERENCE REGIME}

The low interference regime is defined by $a<1$, where $a$ is defined in (4). The probability, $P(a<1)$, depends on the distribution of $r_{c c} / r_{c p}$. Using standard transformation theory [11], some simple but lengthy calculations show that the CDF of $r_{c c} / r_{c p}$ is given by (7). The CDF in (7) can be written as:

$$
P\left(\frac{r_{c c}}{r_{c p}}<x\right)=c_{i 0} x^{-2}+c_{i 1}+c_{i 2} x^{2} \quad i=1,2,3,4,5
$$

where $\Delta=\left(R_{c}^{2}-R_{0}^{2}\right)\left(R_{p}^{2}-R_{0}^{2}\right), c_{10}=0, c_{11}=0, c_{12}=0$, $c_{20}=0.5 R_{0}^{4} / \Delta, c_{21}=-R_{0}^{2} R_{p}^{2} / \Delta, c_{22}=0.5 R_{p}^{4} / \Delta, c_{30}=$ $0.5\left(R_{0}^{4}-R_{c}^{4}\right) / \Delta, c_{31}=R_{p}^{2}\left(R_{c}^{2}-R_{0}^{2}\right) / \Delta, c_{32}=0, c_{40}=$ $-0.5 R_{c}^{4} / \Delta, c_{41}=1+R_{0}^{2} R_{c}^{2} / \Delta, c_{42}=-0.5 R_{0}^{4} / \Delta, c_{50}=0$, $c_{51}=1$ and $c_{52}=0$.

Now $P(a<1)=P\left(a^{2}<1\right)$ can be written as $P(Y<$ $\left.K e^{X} Z^{-\gamma}\right)$ where $Y=|\tilde{f}|^{2} /|\tilde{c}|^{2}, K=N_{p} / N_{c}, X=X_{c c}-$ 


$$
P\left(\frac{r_{c c}}{r_{c p}}<x\right)= \begin{cases}0 & x \leq \frac{R_{0}}{R_{p}} \\ \frac{0.5 x^{2}\left(R_{p}^{2}-R_{0}^{4} x^{-4}\right)-R_{0}^{2}\left(R_{p}^{2}-R_{0}^{2} x^{-2}\right)}{\left(R_{c}^{2}-R_{0}^{2}\right)\left(R_{p}^{2}-R_{0}^{2}\right)} & \frac{R_{0}}{R_{p}}<x \leq \frac{R_{c}}{R_{p}} \\ \frac{0.5\left(R_{c}^{4}-R_{0}^{4}\right)-R_{0}^{2}\left(R_{c}^{2}-R_{0}^{2}\right)+\left(x^{2} R_{p}^{2}-R_{c}^{2}\right)\left(R_{c}^{2}-R_{0}^{2}\right)}{x^{2}\left(R_{c}^{2}-R_{0}^{2}\right)\left(R_{p}^{2}-R_{0}^{2}\right)} & \frac{R_{c}}{R_{p}}<x \leq 1 \\ 1-\frac{0.5 R_{c}^{4} x^{2}+0.5 R_{0}^{4} x^{2}-R_{0}^{2} R_{c}^{2}}{\left(R_{c}^{2}-R_{0}^{2}\right)\left(R_{p}^{2}-R_{0}^{2}\right)} & 1<x \leq \frac{R_{c}}{R_{0}} \\ 1 & x>\frac{R_{c}}{R_{0}}\end{cases}
$$

$X_{c p}$ and $Z=r_{c c} / r_{c p}$. Thus the required probability is:

$$
\begin{aligned}
P\left(Y<K e^{X} Z^{-\gamma}\right) & =P\left(Z<K^{1 / \gamma} e^{X / \gamma} Y^{-1 / \gamma}\right) \\
& =E\left[P\left(Z<K^{1 / \gamma} e^{X / \gamma} Y^{-1 / \gamma} \mid X, Y\right)\right] \\
& =E[P(Z<W \mid W)] \\
& =\int_{0}^{\infty} P(Z<w) f_{W}(w) d w
\end{aligned}
$$

where $W=K^{1 / \gamma} e^{X / \gamma} Y^{-1 / \gamma}$ and $f_{W}($.$) is the PDF of W$. Note that $P(Z<w)$, given in (8), only contains constants and terms involving $w^{ \pm 2}$. Hence, we need the following:

$$
\int_{\theta}^{\kappa} w^{2 m} f_{W}(w) d w=\iint\left(K e^{x} y^{-1}\right)^{2 m / \gamma} f_{X, Y}(x, y) d x d y,
$$

where $m=-1,0,1$ and $f_{X, Y}($.$) is the joint PDF of X, Y$. Now, since $W=K^{1 / \gamma} e^{X / \gamma} Y^{-1 / \gamma}$, the limits $\theta \leq w \leq \kappa$ in (10) imply the following limits for $x$ :

$$
\ln \left(\theta^{\gamma} K^{-1} y\right) \leq x \leq \ln \left(\kappa^{\gamma} K^{-1} y\right) .
$$

Let $\ln \left(\theta^{\gamma} K^{-1} y\right)=A$ and $\ln \left(\kappa^{\gamma} K^{-1} y\right)=B$, then noting that $f_{X, Y}(x, y)=f_{X}(x) f_{Y}(y)$, the integral in (10) becomes:

$$
\begin{aligned}
\int_{\theta}^{\kappa} w^{2 m} f_{W}(w) d w= & \int_{0}^{\infty} K^{2 m / \gamma} y^{-2 m / \gamma} f_{Y}(y) \\
& \times \int_{A}^{B} e^{2 m x / \gamma} f_{X}(x) d x d y .
\end{aligned}
$$

Since $X \sim \mathcal{N}\left(0,2 \sigma_{s f}^{2}\right)$, the inner integral in (11) becomes:

$$
\begin{aligned}
\int_{A}^{B} e^{2 m x / \gamma} f_{X}(x) d x & =\exp \left(\frac{4 m^{2} \sigma_{s f}^{2}}{\gamma^{2}}\right) \\
\times & {\left[\Phi\left(\frac{B-\frac{4 m \sigma_{s f}^{2}}{\gamma}}{\sqrt{2} \sigma_{s f}}\right)-\Phi\left(\frac{A-\frac{4 m \sigma_{s f}^{2}}{\gamma}}{\sqrt{2} \sigma_{s f}}\right)\right], }
\end{aligned}
$$

where $\Phi$ is the CDF of a standard Gaussian. Since $f_{Y}(y)$ is the density function of the ratio of two standard exponentials, it is given by [2]:

$$
f_{Y}(y)=\frac{1}{(1+y)^{2}}, \quad y \geq 0
$$

Using (12) and (13), the total general integral in (10) becomes:

$$
\begin{aligned}
\int_{\theta}^{\kappa} w^{2 m} f_{W}(w) d w= & \int_{0}^{\infty} K^{2 m / \gamma} y^{-2 m / \gamma}(1+y)^{-2} \exp \left(\frac{4 m^{2} \sigma_{s f}^{2}}{\gamma^{2}}\right) \\
& \times\left[\Phi\left(\frac{B-\frac{4 m \sigma_{s f}^{2}}{\gamma}}{\sqrt{2} \sigma_{s f}}\right)-\Phi\left(\frac{A-\frac{4 m \sigma_{s f}^{2}}{\gamma}}{\sqrt{2} \sigma_{s f}}\right)\right] d y \\
& \triangleq I(m, \theta, \kappa) .
\end{aligned}
$$

Substituting (8) and (14) in (9) gives $P(a<1)$ as:

$$
\begin{aligned}
P(a<1) & =P\left(Y<K e^{X} Z^{-\gamma}\right) \\
& =\sum_{i=2}^{5} c_{i 0} I\left(-1, \theta_{i}, \kappa_{i}\right)+c_{i 1} I\left(0, \theta_{i}, \kappa_{i}\right)+c_{i 2} I\left(1, \theta_{i}, \kappa_{i}\right) \\
& =\sum_{i=2}^{5} \sum_{j=0}^{2} c_{i j} I\left(j-1, \theta_{i}, \kappa_{i}\right) .
\end{aligned}
$$

Finally, it can be seen from the limits given in (7) that $\kappa_{i}=\theta_{i+1}$. Hence, the final expression for the probability of occurrence of the low interference regime is:

$$
P(a<1)=\sum_{i=2}^{5} \sum_{j=0}^{2} c_{i j} I\left(j-1, \theta_{i}, \theta_{i+1}\right),
$$

where the $c_{i j}$ were defined after (8), $I\left(j-1, \theta_{i}, \theta_{i+1}\right)$ is given in (14), $\theta_{2}=R_{0} / R_{p}, \theta_{3}=R_{c} / R_{p}, \theta_{4}=1, \theta_{5}=R_{c} / R_{0}$ and $\theta_{6}=\infty$. Hence, $P(a<1)$ can be derived in terms of a single numerical integral. For numerical convenience, (14) is rewritten using the substitution $v=y(y+1)^{-1}$ so that a finite range integral over $0<v<1$ is used for numerical results:

$$
\begin{aligned}
& \int_{\theta}^{\kappa} w^{2 m} f_{W}(w) d w=\int_{0}^{1} K^{2 m / \gamma}\left(\frac{v}{1-v}\right)^{-2 m / \gamma} \exp \left(\frac{4 m^{2} \sigma_{s f}^{2}}{\gamma^{2}}\right) \\
& \times\left[\Phi\left(\frac{B-\frac{4 m \sigma_{s f}^{2}}{\gamma}}{\sqrt{2} \sigma_{s f}}\right)-\Phi\left(\frac{A-\frac{4 m \sigma_{s f}^{2}}{\gamma}}{\sqrt{2} \sigma_{s f}}\right)\right] d v \\
& \triangleq \quad I(m, \theta, \kappa),
\end{aligned}
$$

where $\ln \left(\theta^{\gamma} K^{-1} \frac{v}{1-v}\right)=A$ and $\ln \left(\kappa^{\gamma} K^{-1} \frac{v}{1-v}\right)=B$. Further simplification of (14) appears difficult but the result in (17) is stable and rapid to compute. A comparison of simulated and analytical results is shown in Fig. 2. It can the seen that the analytical formula given in (17) perfectly matches the simulation results. 


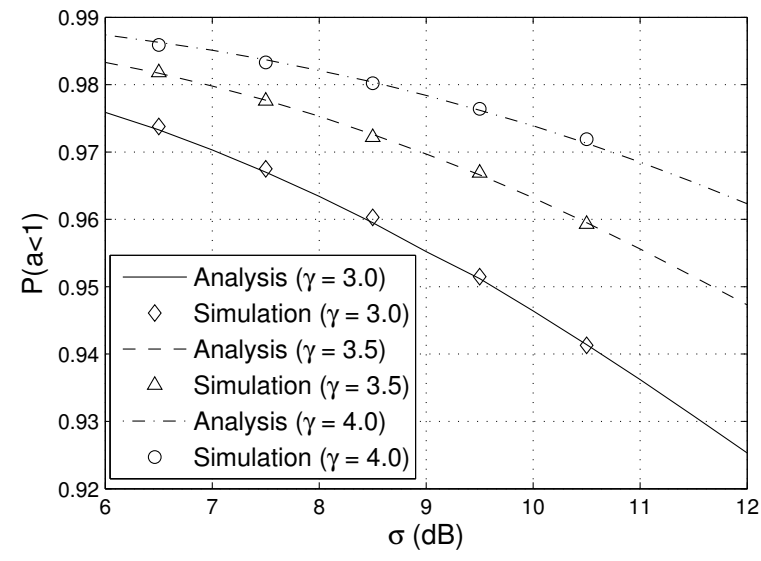

Fig. 2. Probability of occurrence of the low interference regime as a function of shadow fading variance, $\sigma(\mathrm{dB})$. The ratio $R_{p} / R_{c}$ is taken as 10 .

\section{An Approximation For The POWER Loss PARAMETER}

In this section we focus on the power loss parameter, $\alpha$, which governs how much of the transmit power the CR dedicates to relaying the primary message. The exact distribution of $\alpha$ appears to be rather complicated, even for fixed link gains (fixed values of $\Gamma_{c p}, \Gamma_{p c}, \Gamma_{p p}$ and $\Gamma_{c c}$ ). Hence, we consider an extremely simple approximation based on the idea that $|s| \times|t|$ is usually small and $|s| \times|t|>>|t|$. This approximation is motivated by the fact that the $\mathrm{CP}$ link is usually very weak compared to the PP link. This is because the CRs will employ much lower transmit powers than the PU. With this assumption it follows that $|t|^{2}\left(1+|s|^{2}\right)$ is small and we have:

$$
\begin{aligned}
\sqrt{\alpha} & =\frac{|s|}{|t|}\left[\frac{\left(1+|t|^{2}\left(1+|s|^{2}\right)\right)^{1 / 2}-1}{1+|s|^{2}}\right] \\
& \approx \frac{|s|}{|t|}\left[\frac{1 / 2|t|^{2}\left(1+|s|^{2}\right)}{1+|s|^{2}}\right] \\
& =\frac{|s||t|}{2} \\
& =\sqrt{\alpha_{\text {approx }}} .
\end{aligned}
$$

Expanding $\alpha_{\text {approx }}$ we have:

$$
\alpha_{\text {approx }}=\frac{A_{p} A_{c} P_{p} P_{c}}{4 N_{p}^{2}} e^{\left(X_{p p}+X_{c p}\right)} r_{p p}^{-\gamma} r_{c p}^{-\gamma}|\tilde{p}|^{2}|\tilde{f}|^{2} \text {. }
$$

This approximation is very effective for low values of $\alpha_{\text {approx }}$, but is poor for larger values since $\alpha_{\text {approx }}$ is unbounded whereas $0<\alpha<1$. To improve the approximation, we use the conditional distribution of $\alpha_{\text {approx }}$ given that $\alpha_{\text {approx }}<1$. This conditional variable is denoted, $\hat{\alpha}$. The exact distribution of $\hat{\alpha}$ is difficult for variable link gains. However, the approximation has a simple representation which leads to considerable insight into the power loss and how it relates to system parameters. For example $\alpha_{\text {approx }}$ is proportional to $|s|^{2}|t|^{2}$ so that high power loss may be caused by high values of $|s|$ or $|t|$ or moderate values of both. Now $|s|$ and $|t|$ relate to the $\mathrm{PP}$ and $\mathrm{CP}$ links respectively. Hence the $\mathrm{CR}$ is forced to use high power relaying the PU message when the CP link is strong. This is obvious as the relay action needs to make up for the strong interference caused by the $\mathrm{CR}$. The second scenario is that the $\mathrm{CR}$ has high $\alpha$ when the PP link is strong. This is less obvious, but here the PU rate is high and a substantial relaying effort is required to counteract the efforts of interference on a high rate link. This is discussed further in Section V. It is worth noting that the condition $|s||t|>>|t|$ holds good only for some specific values of channel parameters. Hence, although it is motivated by a sensible physical scenario, it certainly needs checking. Results in Figs. 3, 5 and 6 show that it works very well.

For fixed link gains, the distribution of $\hat{\alpha}$ is:

$$
\begin{aligned}
P\left(\alpha_{\text {approx }}<x \mid \alpha_{\text {approx }}<1\right) & =P(\hat{\alpha}<x) \\
& =\frac{P\left(\alpha_{\text {approx }}<x\right)}{P\left(\alpha_{\text {approx }}<1\right)} .
\end{aligned}
$$

Thus, to compute the distribution function of $\hat{\alpha}$ we need to determine $P\left(\alpha_{\text {approx }}<x\right)$ which can be written as

$$
P\left(\alpha_{\text {approx }}<x\right)=P\left(|s|^{2}|t|^{2}<4 x\right) \text {. }
$$

In the analytical approximation below we assume that $|s|^{2}$ and $|t|^{2}$ are exponential, i.e., we ignore the conditioning on $a<1$. The conditioning can be handled exactly but results suggest that a simple exponential approximation is satisfactory. Let $E\left(|s|^{2}\right)=\mu_{s}, E\left(|t|^{2}\right)=\mu_{t}$ with $\mu_{s}=P_{p} \Gamma_{p p} / N_{p}$ and $\mu_{t}=P_{c} \Gamma_{c p} / N_{p}$. Further, suppose that $U$ and $V$ represent i.i.d. standard exponentials, then we have

$$
\begin{aligned}
P\left(\alpha_{\text {approx }}<x\right) & =P\left(U V<\frac{4 x}{\mu_{s} \mu_{t}}\right) \\
& =E_{V}\left(P\left(U<\frac{4 x}{V \mu_{s} \mu_{t}}\right)\right) \\
& =E_{V}\left(1-\exp \left(\frac{-4 x}{V \mu_{s} \mu_{t}}\right)\right) \\
& =1-\int_{0}^{\infty} \exp \left(\frac{-4 x}{v \mu_{s} \mu_{t}}-v\right) d v \\
& =1-\sqrt{\frac{16 x}{\mu_{s} \mu_{t}}} K_{1}\left(\sqrt{\frac{16 x}{\mu_{s} \mu_{t}}}\right),
\end{aligned}
$$

where $K_{1}($.$) represents the modified Bessel function of the$ second kind and the integral in (22) can be found in [12]. Using the expression given in (22), the CDF of $\hat{\alpha}$ follows from (20). Note that the CDF of $R_{c}$ can easily be obtained in the form of a single numerical integral for fixed powers.

\section{RESUlts}

In the results section, the default parameters are $\sigma=8$ $\mathrm{dB}, \gamma=3.5, R_{0}=1, R_{c}=100 \mathrm{~m}, R_{p}=1000 \mathrm{~m}$ and $N_{p}=N_{c}=P_{p}=P_{c}=1$. The parameter $A_{p}$ is determined by ensuring that the link PP has an SNR $\geq 5 \mathrm{~dB} 95 \%$ of the time in the absence of any interference. Similarly, assuming that both PU and $\mathrm{CR}$ devices have same threshold power at their cell edges, the constant $A_{c}=A_{p}\left(R_{p} / R_{c}\right)^{-\gamma}$. Unless otherwise stated these parameters are used in the following.

\section{A. Low interference regime}

In Fig. 2 we show that the low interference regime, $a<1$, is the dominant scenario. For typical values of $\gamma \in[3,4]$ and 


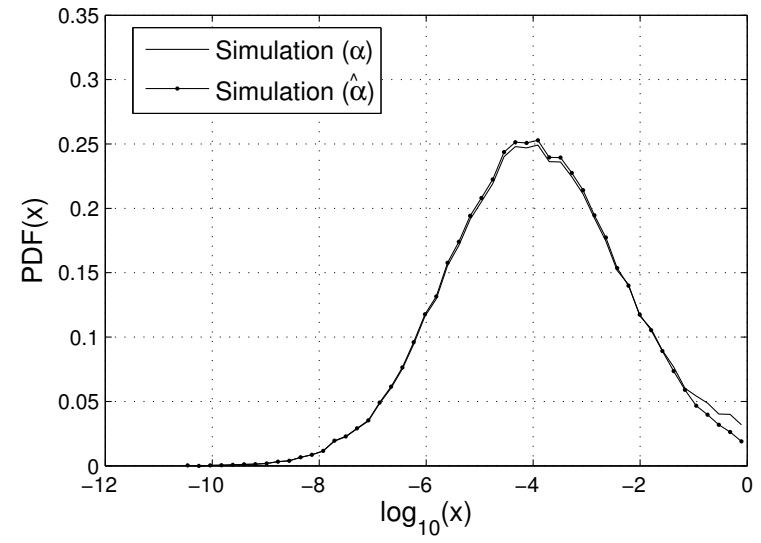

Fig. 3. PDFs of $\log _{10}(\alpha)$ and its approximation $\log _{10}(\hat{\alpha})$.

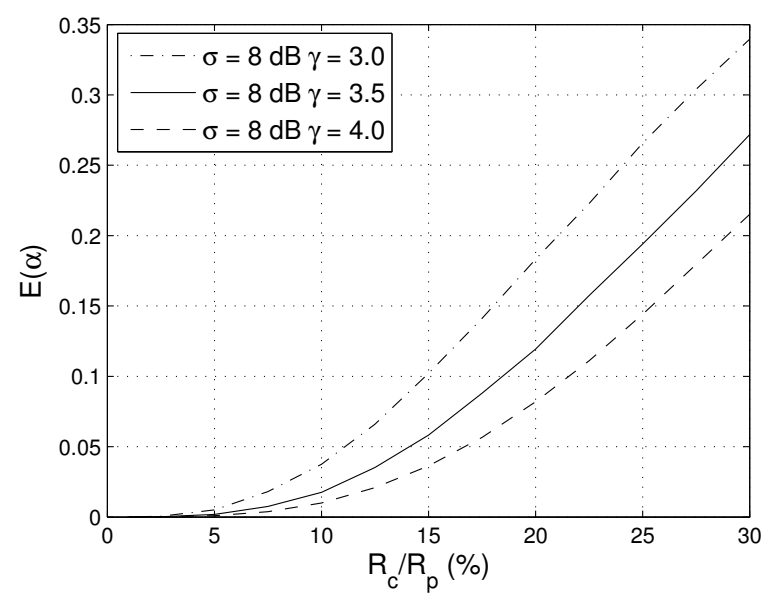

Fig. 4. Mean value of the power loss parameter, $\alpha$, as a function of the ratio $\frac{R_{c}}{R_{p}}$.

$\sigma \in[6,12] \mathrm{dB}$ we find that $P(a<1)$ is usually well over $90 \%$. Figure 2 also verifies the analytical result in (15).

The relationship between $P(a<1)$ and the system parameters is easily seen from (4) which contains the term $\left(r_{c c} / r_{c p}\right)^{\gamma / 2} \exp \left(\left(X_{c c}-X_{c p}\right) / 2\right)$. When $R_{c}<<R_{p}$, this term decreases dramatically as $\gamma$ increases and as $\sigma$ increases the term increases. Also, as $R_{c}$ increases $r_{c c} / r_{c p}$ tends to increase which in turn increases $P(a<1)$. When $R_{c} \approx R_{p}$ the low and high interference scenarios occur with similar frequency. This may be a relevant system consideration if CRs were to be introduced in cellular bands where the cellular hot spots, indoor micro-cells and CRs will have roughly the same coverage radius. Note that $a$ is independent of the transmit power, $P_{c}$. These conclusions are all verified by simulations which are omitted for reasons of space.

\section{B. Statistics of the power loss parameter, $\alpha$}

Figures 3-5 all focus on the properties of $\alpha$. Figure 3 shows that the probability density function (PDF) of $\alpha$ is extremely well approximated by the PDF of $\hat{\alpha}$. In Fig. 4 we see that $E(\alpha)$ increases with increasing values of $R_{c} / R_{p}$ and decreasing values of $\gamma$. This can be seen from (19) where $\alpha_{a p p r o x}$

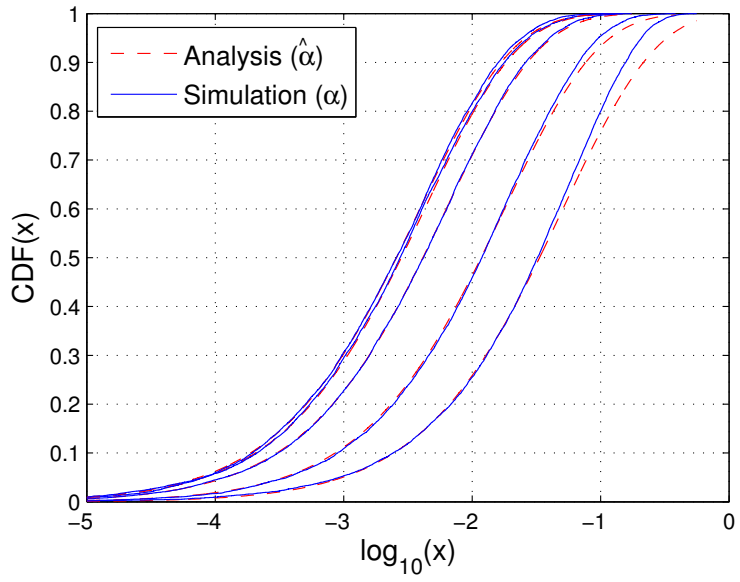

Fig. 5. Comparison of the exact and analytical CDFs of the power loss factor on a logarithmic scale for fixed link gains. Results are shown for 5 drops.

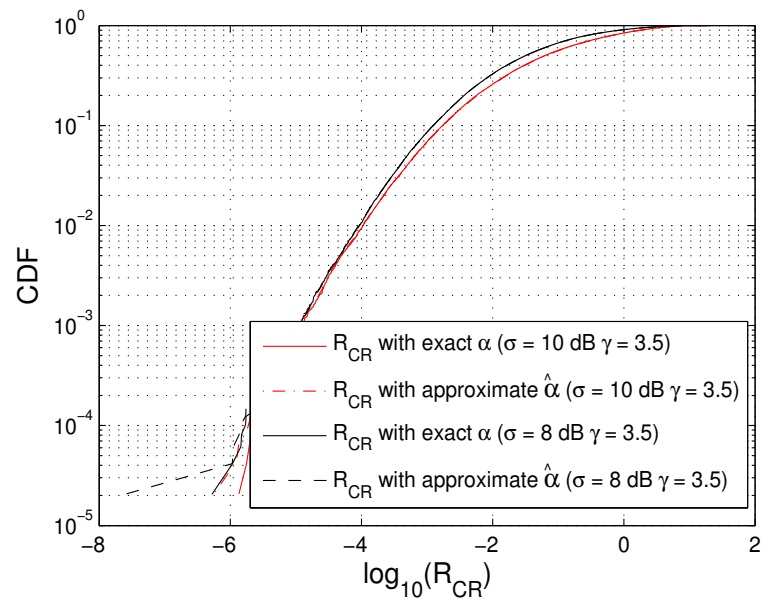

Fig. 6. $\mathrm{CDF}$ of the $\mathrm{CR}$ rates with the exact $\alpha$ and the approximate $\hat{\alpha}$.

contains a $\left(r_{p p} r_{c p}\right)^{-\gamma}$ term which increases as $\gamma$ decreases. The increase of $E(\alpha)$ with $R_{c}$ follows from the corresponding increase in $P_{c}$ to cater for larger $R_{c}$ values. In Fig. 4 we have limited $R_{c} / R_{p}$ to a maximum of $30 \%$ as beyond this value the high interference regime is also present with a nonnegligible probability. In Fig. 5 we see the analytical CDF in (22) verified by simulations for five different scenarios of fixed link gains (simply the first five simulated values of $\Gamma_{p p}$ and $\left.\Gamma_{c p}\right)$. Note that in the different curves each correspond to a random drop of the PU and CR transmitters. This fixes the distance and shadow fading terms in the link gains in (2), thereby the remaining variation in (1) is only Rayleigh. By computing a large number of such CDFs and averaging them over the link gains a single CDF can be constructed. This approach can be used to find the PDF of $\hat{\alpha}$ as shown in Fig. 3. Note that the curves in Fig. 5 do not match exactly since the analysis is for $\hat{\alpha}$ and the simulation is for $\alpha$.

\section{CR rates}

Figures 6-8 focus on the CR rate $R_{C R}$. Figure 6 demonstrates that the use of $\hat{\alpha}$ is not only accurate for $\alpha$ but also leads to excellent agreement for the $\mathrm{CR}$ rate, $R_{C R}$. 


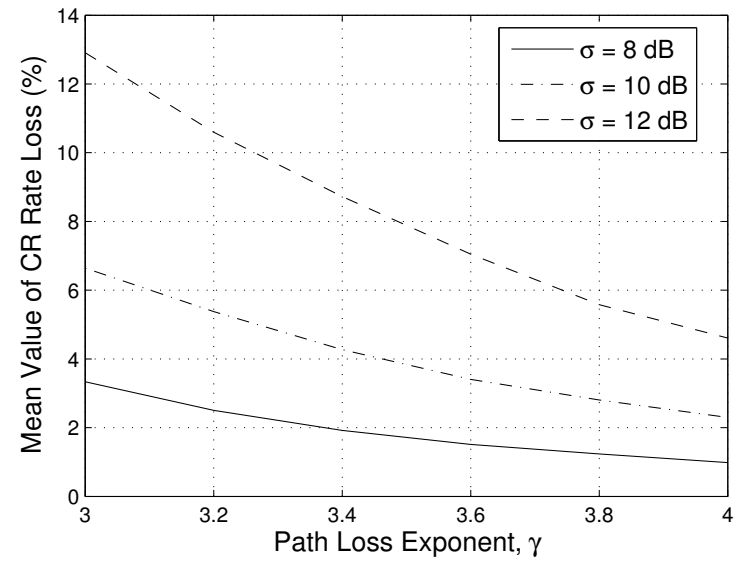

Fig. 7. Mean value of the CR rate loss as a function of $\gamma$.

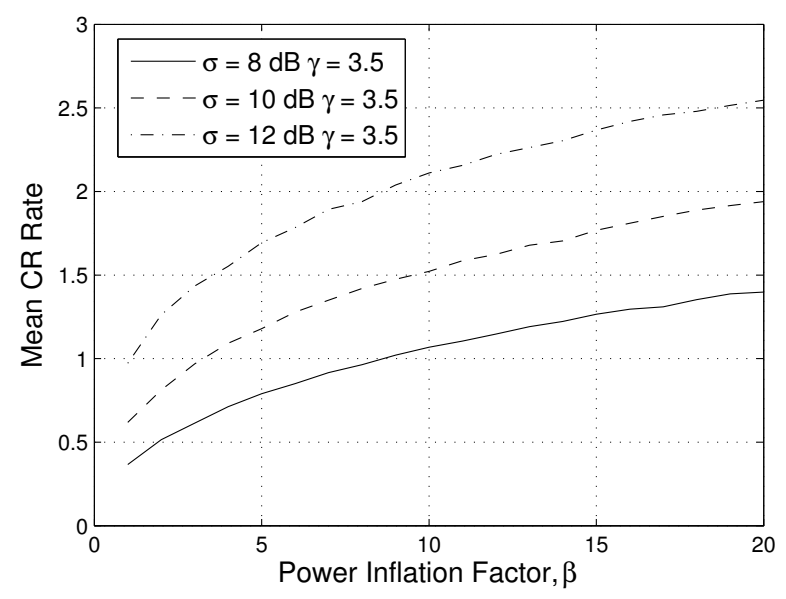

Fig. 8. Variation of the mean CR rate with the power inflation factor, $\beta$.

This agreement holds over the whole range and for all typical parameter values. Figure 7 shows the $\%$ loss given by $\left[R_{C R}(\alpha=0)-R_{C R}(\alpha)\right] /\left[R_{C R}(\alpha=0)\right] \%$. The loss decreases as $\gamma$ increases, as discussed above, and increases with $\sigma$. From (19) it is clear that increasing $\sigma$ lends to larger values of $\exp \left(X_{p p}+X_{c p}\right)$ which in turn increases $\alpha$ and the rate loss. Note that the rate loss is minor for $\sigma \in[8-10] \mathrm{dB}$ with $R_{c}=R_{p} / 10$. In a companion paper [13], we show that the interference to the PU increases with $\sigma$ and decreases with $\gamma$. These results reinforce this observation, i.e., when the PU suffers more interference ( $\sigma$ is larger) the $\mathrm{CR}$ has to devote a higher part of its power to the PU. Consequently the percentage rate loss is higher.

Finally, in Fig. 8 we investigate the gains available to the $\mathrm{CR}$ through increasing transmit power. The original transmit power, $P_{c}$, is scaled by $\beta$ and the mean CR rate is simulated over a range of $\beta$ values. Due to the relaying performed by the $\mathrm{CR}$, the PU rate is unaffected by the CR for any values of $\beta$ and so the $\mathrm{CR}$ is able to boost its own rate with higher transmit power. Clearly the increased value of $\alpha$ for higher values of $\beta$ is outweighed by the larger $P_{c}$ value and so the $\mathrm{CR}$ does achieve an overall rate gain. In a very coarse way these results suggest that multiple CRs may be able to co-exist with the PU since the increased interference power might be due to several CRs and the rate gain might be spread over several CRs. Of course, this conclusion is speculative as the analysis is only valid for a single CR.

\section{CONCLUSION}

In this paper we derive the probability that the "low interference regime" holds and demonstrate the conditions under which this is the dominant scenario. We show that the probability of the low interference regime is significantly influenced by the system geometry. When the CR coverage radius is small relative to the $\mathrm{PU}$ radius, the low interference regime is dominant. When the $\mathrm{CR}$ coverage radius approaches a value similar to the PU coverage radius, the low and high interference regimes both occur with roughly equal probability. In addition we have derived a simple, accurate approximation to $\alpha$ which gives considerable insight into the system capacity. The $\alpha$ approximation shows that CR rates are reduced by large CR coverage zones, small values of $\gamma$ and large values of $\sigma$. Finally, we have shown that the CR can increase its own rate with higher transmit powers, although the relationship is only slowly increasing as expected.

\section{REFERENCES}

[1] A. Ghasemi and E. S. Sousa, "Spectrum sensing in cognitive radio networks: requirements, challenges and design trade-offs [cognitive radio communications]," IEEE Communications Magazine, vol. 46, no. 4, pp. 32-39, April 2008

[2] - "Fundamental limits of spectrum-sharing in fading environments," IEEE Transactions on Wireless Communications, vol. 6, no. 2, pp. 649658, Feb. 2007.

[3] N. Devroye, P. Mitran, and V. Tarokh, "Achievable rates in cognitive radio channels," IEEE Transactions on Information Theory, vol. 52, no. 5, pp. 1813-1827, May 2006.

[4] I. Maric, A. Goldsmith, G. Kramer, and S. S. (Shitz), "On the capacity of interference channels with one cooperating transmitter," European Transactions on Telecommunications, vol. 19, pp. 405-420, Apr. 2008.

[5] A. Jovicic and P. Viswanath, "Cognitive radio: An information-theoretic perspective," in Proc. IEEE International Symposium on Information Theory, July 2006, pp. 2413-2417.

[6] J. Jiang and Y. Xin, "A new achievable rate region for the cognitive radio channel," in Proc. IEEE International Conference on Communications, May 2008, pp. 1055-1059.

[7] G. Kramer, "Review of rate regions for interference channels," in Proc. International Zurich Seminar on Communications, 2006, pp. 162-165.

[8] M. Costa, "Writing on dirty paper (corresp.)," IEEE Transactions on Information Theory, vol. 29, no. 3, pp. 439-441, May 1983.

[9] W. Wu, S. Vishwanath, and A. Arapostathis, "Capacity of a class of cognitive radio channels: Interference channels with degraded message sets," IEEE Transactions on Information Theory, vol. 53, no. 11, pp. 4391-4399, Nov. 2007.

[10] M. Vu, N. Devroye, and V. Tarokh, "On the primary exclusive regions in cognitive networks," IEEE Transactions on Wireless Communications, accepted.

[11] A. Papoulis and S. U. Pillai, Probability, Random Variables and Stochastic Processes, 4th ed. New York: McGraw Hill, 2002.

[12] I. S. Gradshteyn and I. M. Ryzhik, Table of Integrals, Series, and Products, 7th ed. California USA: Academic Press, 2007.

[13] M. F. Hanif, M. Shafi, P. J. Smith, and P. Dmochowski, "Interference and deployment issues for cognitive radio systems in shadowing environments," to appear in Proc. IEEE ICC, June 2009. 